\title{
Nature of Bruxism
}

\author{
Radostina Vasileva ${ }^{1}$
}

\section{Department of Dental Material Science and Propaedeutic of Prosthetic Dental Medicine, Faculty of Dental Medicine, Medical University Varna;}

\begin{abstract}
Due to practical constraints on the study of the daily events of bruxism, the clinical trials were mainly focused on the nature of nightly bruxism. This mandibular parafunctional behavior that occurs during the sleeping hours consists in rhythmically often strong tooth friction or prolonged squeeze by muscle abbreviation.

The feature of the phenomenon is multi-layered. The proper knowledge and detection of the early symptoms are the keys for the treatment of bruxism.
\end{abstract}

Keywords: treatment, bruxism, squeeze, paraffin, occlusal forces

\section{Introduction}

Permanent clenching of the teeth can occur at the maximal intercuspidation, and also in an eccentric occlusal position such as anterior incisal edge to incisal edge, the top of the canine to the top of the same or functionality of the rear groups. The rhythmic movements of the lower jaw at night bruxism reflect typical episodes that may emerge through interrupted intervals.(1)

A study provides an indication of an average of 25 episodic manifestations of bruxism for one night. Although each episodic event may go from 8-9 seconds and according to a study there are cases of episodic duration up to 5 minutes. The average total time for bruxism during the sleeping period is $42 \mathrm{~s}$, but according to the scientific literature there were cases with 162 minutes duration.(2.3) Also, the studies show a variety of the manifestations of bruxism for the same person in different nights. During the mandibular parafunctional activity such as bruxis, could be generated excessive forces for long periods of time that exceed approximately 20-minutes functional contact of the teeth when swallowing and chewing for a 24-hour period. 
Nightly Bruxism can generate huge occlusal forces that sometimes can be considerably larger than the individual's conscious efforts when he does not sleep. A study shows that more than $65 \%$ of the nightly episodic events of the Bruxism exceeds the forces generated by the chewing process. As a result, the teeth, the alveolar and periodontal support apparatus, and the temporomandibular joints undertake significant stresses. Like comparison, the average workforce that a natural tooth can use is about 175 psi, while manifestations of bruxism may increase this force to $300 \mathrm{psi}$ and in isolated cases at 100000psi to 175000psi.(4) Also, mandibular functions can generate tooth contact forces of about 17,200lb-sec per day, while the paraoperative activity can generate about 57,600 per day. It is possible that the cortical inhibitors with normal functionning in awaken state to be suppressed during sleep and thus allow the chewing muscles to exert much more occlusal forces.(5)

Usually the mandibular relationship is stable at central occlusion with full dental composition, so the direction of the functional strength is vertical and well tolerated. The direction of the parafunctional forces friction is horizontal and not well tolerated, because mandibular eccentric movements are included. The chewing machine is more exposed to the destructive consequences of the forces of dental contact in bruxism, than to the forces generated by functional teeth contact. In the structures of the chewing machine are affected, in general, by the forces of Bruxism, the supporting tissues, the associated muscles and temporomandibular joints. The general resistance of each structure together with the duration, the frequency and the strength of the mandibular parafunctional manifestations are of a huge importance for the ultimate effect. Hence, although the forces generated by the parafunctional mandibular activity are transmitted to the structures of the chewing machine, for some forces the destructive effect may be missing due to their consumption, while for others violations of varying degrees could exist.(6)

In the clinical symptoms of Bruxism, intra and extra oral changes could be differed. Extraoral changes include the hypertrophy of muscle masseter and the altered bite height. In the case of hypertrophy, as a result of the frequent muscle contractions that the patient performs, a muscle thickening could be obtained. In case of change in the height of the bite, we could observe chin prominence, drop of the nose, change of the nasolabial folds - old age appearence.(7)

In 1965, Powell and Zander found that people without bruxism make 260 tooth contacts for 8 hours of sleep. For the people with bruxism, these contacts are from 1,200 to 12,000 in 8 hours. Data shows that an opportunity for severe occlusal trauma with all of the following complications is available (8.9). The centric bruxism is characterised by clamping of teeth in a static position - without sliding movements. This type of bruxism provokes weaker dental erosions, but with more muscle disorders. The tinglings and the pain of tooth, the periodontium and the joint are frequent. The centric bruxism is the heaviest shape with regard to the joint, muscles and periodontium damages. This kind of bruxism is the basis for severe periodontal disease and dental mobility. There is also a common pain from warm and cold. Of particular interest are the fractures of the teeth, which may be manifested from mild dental fractures to severe cleavage of the tooth in two. A major symptom of the centric bruxism is the formation of hypertrophied bone substance - torus, placed lingually or vestibularly on the tooth ranges. The muscles are particularly sensitive and as a result of their severe hypertrophy, it is possible to squeeze the salivary gland with a pronounced symptom of xerotomy.(10.11.12)

The sensitivity during palpation of the chewing muscles may be a common symptom of Bruxism, as well as complaints of a stiffness of chewing muscles or restricted lower jaw movements while awaking; a headache as a result of muscle tension. It is possible that the parafunctional manifestations of the temporomandibular 
joint and especially in cases of compromise with the health of the surrounding soft tissues, such as the behind disc tissue, sleeping with his shoulder or arm, the individual can exert pressure on the lower jaw so that the contralateral side condyle could be shifted to lateral - backward direction, which leads to compression of the behind disc tissue, as well as on the side additional ligament. In this case, it can be exerted an excessive pressure on the tissue, a synovial membrane. So that, the sensitivity of the affected joint may increase due to the presence of peripheric receptors for pain in these tissues and the behind disc tissue may have accompanied edematous inflammatory reaction. This would lead to a frontal medial movement of the affected condition. (13.14.15)

In case of unilateral inflammation of the behind disc tissue, usually the patient reports the feeling of presence of the rear open bite from the homolateral side of the affected joint, as well as a larger occlusal contact in the area of the side incisiros on the opposite side. In case of double-sided inflamed behind disc tissues, the patient gives information about rear double - sided open bite, as well as for a larger occlusal contact in the area of the central incisors.(16.17.18) The continuous bruxism, without simultaneous application of an antiinflammatory treatment and an intravascular bus, contributes to the development of inflammatory processes in the tissues and slows down the healing process. The remodeling or the osteoarthritis of the functional surfaces of the temporomandibular joint may be perceived as an adaptive process of change in response to mechanical factors and the functional needs. As already mentioned, the recurrent overload of the temporomandibular joint, which may occur in bruxism, is often accepted as a cause of osteoarthritis of the joint.(19.20.21)

It is also supposed that the Bruxism and particularly the crushing of the front teeth may lead to internal relaxation of the temporomandibular joint. In case of tip-to-tip contact, when the lower jaw is eccentric, the articular disk of the homolateral temporomandibular joint becomes embraced in a definite location from the upper abdomen of the pterygoideus lateralis muscle. At the same time, the head of the condyle moves in the side direction and for some individuals sideways backwards, so there is a potential possibility of additional tension on the side ligament of the joint capsule. If this ligament structure is exposed to a risk, the result could be a disk shift.(22)

The scientific research shows that during sleep the skeletal muscles activate themselves, which results in the body movement. As the dental system consists of skeletal muscles, the fact that the research studies the track of the movements of the lower jaw together with the other body movements during sleep is not surprising. In fact, the lower jaw may be considered as a limb because the temporomandibular joints are hinged, similar to those of the upper and the lower limbs. By the relationship between the manifestations of the bruxism and the different periods of sleep, there are reports of the occurrence of nightly mandibular parafunctional manifestations in all periods of sleep, despite the presence of information that bruxism occurs when the individual moves from the period of deeper to lighter sleep, as well as in the period characterized by rapid eye movement.(REM) The sound of clenching is quite strong and can be disturbing for the others around.(23)

Bone resorption and pathological bone pockets could be diagnosed by x-ray. In another form - the eccentric bruxism exists with forward, back, left and right movements, wchich means slide movements, so it causes stronger abrasion of the teeth, but with subsequent muscle relaxation, it becomes a periodontium. The most important symptom is the erasure of the teeth chewing surface. The most frequent clinical consequences of bruxism trauma is the teeth wear and tear that affects the local general occlusal or incisal surfaces, who can be from light to heavy. The strong wear and tear of the front six teeth can lead to both aesthetic problems 
of the patient as well as difficulties in recovery from a dentist and especially in the case of parallel general wear and tear of the rear, that can lead to reduction of the vertical facial size or a tight closing of the bottom jaw. Interproximal diastheme and a food retention can also be a result of the severe back teeth wear. To the aesthetic problem we can add other consequences of the bruxism related to the teeth and supporting the tissues as follows: high heat sensitivity, over-mobility, periodontal ligament injury and periodontium, hypercytosis, broken tips, pulpitis and pulp necrosis. As a result of this erasure their sensitivity increases and there is a possibility to affect the pulp. In advanced forms of eccentric bruxism, the height of the bite decreases. Patients feel twitching, tingling in the gums, with a general discomfort and a headache. Bone glands and exostoses could be observed by x-ray. The eccentric form of bruxism leads to severe abrasion with all subsequent disabilities - respectively increased muscular strength, increased chewing time and various sliding movements, which on its turn, deepens the process. All these disorders badly damage the maxillofacial area and therefore must be promptly diagnosed, avoided and treated. (24)

The Diagnosis of Bruxism is relatively easy in case of an accurate anamnesis, examination and x-ray examination. The serrated side edges of the tongue are considered as an indication of teeth clenching, although the scientific literature does not provide any further evidence to support this statement. Other clinical signs of bruxism observed by the dentist are: occlusal and incisal teeth wear and tear, mobility of teeth affected by periodontitis, tooth fractures, hypertrophy of muscle masseter, as well as hypertrophy of the forehead temporal muscle, expansion of the place occupied by the periodontal ligament, as well as the ligament itself, which is demonstrated in radiography and a sensitivity of chewing muscles during palpation. Symptoms of bruxism for which the patient can give information are: a sense of stiffness of the chewing muscles that occur when waking, although the daily squeezing can cause the same sensation, myalgia, mofacial pain or both, over-sensitivity of the teeth and sounds formed by the friction of the teeth during sleep (information given by the husband or other people).

The establishing of nightly or daily bruxism as a cause of the symptoms of the patient is easier when he realizes that they lead to this type of jaw parafunctional activity. As about $20 \%$ of the patients report to grip of the teeth while waking and about $10 \%$ during sleep, as well as $6-12 \%$ for tooth friction during sleep, it is difficult to do the conclusion that bruxism or other jaw parafunctional activity lead to the observed symptoms. To conclude that the nightly bruxism is the main factor, the dentist must rely on information of the patient for the sensitivity of the joints or the muscles, muscle weaknesses or stiffness of the chewing muscles when waking up. Although that these symptoms are usually more prominent when waking up, the dentist must be aware of that they may also be due to the later sensitivity of chewing muscles as in the case of overloading muscles anywhere else in the human body. The most obvious sign of nightly bruxism is the erasure of the cutting edges of the front teeth and at the occlusal surface of the rear teeth. The dentist must distinguish the teeth wear caused during a normal chewing process or personal habits and the wear due to friction of opposing teeth in case of bruxism. The first shows typical wear of non-touching tooth surfaces, while in the second one there is teeth touch. Many patients could believe that their tooth erasure is due to food, but the west way of nutrition would not lead to such a phenomenon. The prevention of bruxism includes the acceptance of enough minerals and vitamins, avoiding alcohol, caffeine and drugs. There is a good effect from exercises for peace and relaxation of the muscles and the joints. Unfortunately, once started this disease is difficult to pass without specialized help and treatment. 


\section{Conclusion}

The knowing and the accurate diagnosis of Bruxism are extremely important. Manifestations such as erased tooth surfaces, muscle hypertrophy, facial deformity, bone enlargement, wedge-shaped defects, joints and muscles pain are a sure sign of the presence of a parafunction. After the proper identification of normal tooth erasure and a pathological one, it is necessary to proceed with an early treatment.

\section{References}

1. Baba K, Clark GT, Watanabe T, Ohyama T. Bruxism force detection by a piezoelectric film-based recording device in sleeping humans. J Orofac Pain. 2003;17:58-64.

2. Baba K, Haketa T, Clark GT, Ohyama T. Does tooth wear status predict ongoing sleep bruxism in 30-year-old Japanese subjects? Int J Prosthodont. 2004;17:39-44.

3. Butler JH. Occlusal adjustment. Dent Dig. 1970;76:422-426.

4. Ekfeldt A, Hugoson A, Bergendal T, Helkimo A. An individual tooth wear index and an analysis of factors correlated to incisal and occlusal wear in an adult Swedish population. Acta Odontol Scand. 1990;48:343-349.

5. Frumker SC. Occlusion and muscle tension. Basal facts. 1981;4:85-87.

6. Holmgren K, Sheikholeslam A, Riise C. Effect of a full-arch maxillary occlusal splint on parafunctional activity during sleep in patients with nocturnal bruxism and signs and symptoms of craniomandibular disorders. J Prosthet Dent. 1993;69:293-297

7. Ikeda T, Nishigawa K. Criteria for the detection of sleep associated bruxism in humans. J Orofac Pain. 1996;10:270-282.

8. Jadidi F, Castrillon E, Svensson P. Effect of conditioning electrical stimuli on temporalis electromyographic activity during sleep. J Oral Rehabil. 2007;34:152-159.

9. Johansson A, Johansson AK, Omer R, Carlsson GE. Rehabilitation of the worn dentition. J Oral Rehabil. 2008;35:548-56

10. Kato T, Thie NM, Huynh N, Miyawaki S, Lavigne GJ. Topical review: sleep bruxism and the role of peripheral sensory influences. J Orofac Pain. 2003;17:191-213

11. Kato T, Thie NM, Montplaisir JY, Lavigne GJ. Bruxism and orofacial movements during sleep. Dent Clin N Am. 2001;45:657-684.

12. Knight DJ, Leroux BG, Zhu C, Almond J, Ramsay DS. A longitudinal study of tooth wear in orthodontically treated patients. Am J Orthod Dentofac Orthop. 1997;112:194-202.

13. Korioth TW, Bohlig KG, Anderson GC. Digital assessment of occlusal wear patterns on occlusal stabilization splints: a pilot study. J Prosthet Dent. 1998;80:209-213

14. Koyano K, et al. Assessment of Bruxism in the clinic. J Oral Rehab. 2008;35:495-508

15. Lavigne GJ, Khoury S, Abe S, Yamaguchi T, Raphael K. Bruxism physiology and pathology: an overview for clinicians. J Oral Rehabil. 2008;35:476-494

16. Lavigne GJ, Khoury S, Abe S, Yamaguchi T, Raphael K. Drugs and bruxism: a critical review. J Orofac Pain. 2003;17:99-111

17. Lobbezoo F, et al. Principles of Maganement of Bruxism. J Oral Rehab. 2008;35:509-52350.

18. Lobbezoo F, Zaag J, Selms MKA, Hamburger HL, Naeije M. Principles for the management of bruxism. J Oral Rehabil. 2008;35:509-523

19. Minakuchi H, Clark GT (2004) The sensitivity and specificity of miniature bruxism detection device. J Dent Res 83(special issue A)

20. Pierce CJ, Gale EN. Methodological considerations concerning the use of bruxcore plates to evaluate nocturnal bruxism. J Dent Res. 1989;68:1110-1114

21. Pintado MR, Anderson GC, Long R, Douglas WH. Variation in tooth wear in young adults over a two-year period. J Prosthet Dent. 1997;77:313-320.

22. Rugh JD, Solberg WK. Electromyographic studies of bruxist behavior before and during treatment. J Calif Dent Assoc. 1975;3:56-59. 
23. Takeuchi H, Ikeda T, Clark GT. A piezoelectric film-based intrasplint detection method for bruxism. J Prosthet Dent. 2001;86:195-202

24. Velly-Miguel AM, Montplaisir J, Rompre PH, Lund JP, Lavigne GL. Bruxism and other orofacial movements during sleep. J Craniomandib Dis Fac Oral Pain. 1992;6:71-81.

\section{Corresponding author:}

Radostina Vasileva,

Department of Dental Material Science

and Propaedeutics of Prosthetic Dental Medicine,

Faculty of Dental Medicine,

Medical University Varna,

email: radost11dent@abv.bg 